\title{
Parkinson's Disease and Software: The Applicability Challenge
}

\author{
Nabil Aly ${ }^{1 *}$ and Maththew Mcnamee ${ }^{2}$ \\ ${ }^{1}$ Consultant Physician, University Hospital Aintree, Liverpool-UK \\ ${ }^{2}$ Specialist Registrar, University Hospital Aintree, Liverpool-UK
}

*Corresponding author: N Aly, Consultant physician, University Hospital Aintree, Liverpool, Lower Lane, Liverpool L9 7AL, UK

To Cite This Article: Nabil Aly, Maththew M. Parkinson's Disease and Software: The Applicability Challenge. Am J Biomed Sci \& Res. 2019 - 1(5). AJBSR.MS.ID.000548. DOI: 10.34297/AJBSR.2019.01.000548

Received: February 28, 2019 | Published: March 11, 2019

\section{Introduction}

Parkinson's disease (PD) is a progressive neurodegenerative movement disease affecting over 5 million people worldwide. However, there is currently no definitive test for PD by nonspecialist clinicians, especially in the early disease stages where the symptoms may be subtle and poorly characterized. Subsequently, the lack of definitive test for early PD results in a high misdiagnosis rate (up to $25 \%$ by non-specialists) and people can have the disease for many years before being diagnosed and managed. Undoubtedly, there is a need for a more accurate, objective means of early detection, ideally one which can be used by individuals in their home setting. In addition, objective measurements of motor signs are of vital importance for monitoring and developing disease modifying therapies, particularly for the early stages of the disease when potential neuroprotective treatments could stop neurodegeneration.

Digital technologies for objectively quantifying PD motor sign do exist and more new ones are being developed. One of the most frequently used technologies is finger tapping, where patients are asked to intermittently press buttons as fast as possible for a given time. More recently, wearable inertia measurement units (IMUs- multiple sensors are applied on various areas of subject's body during task performance) have been employed to measure information about gait, posture, tremor, bradykinesia and even dyskinesia (involuntary irregular movements) [1]. IMUs can be uploaded to modern smartphones, which has encouraged attempts to combine finger tapping and IMUs in a single device.

\section{Early Diagnosis}

The routine interaction with computer keyboards can be used to detect motor signs in the early stages of PD. Software has been developed in order to measure the key hold times (HT-the time required to press and release a key) during the normal use of a computer without any change in hardware and converts it to a PD motor index. This is achieved by the automatic discovery of patterns in the time series of key hold times using an ensemble novel regression algorithm, the numerical neuro QWERTY index (nQi). This new approach can discriminate early PD groups from controls and the performance was comparable or better than two other quantitative motor performance tests used clinically: alternating finger tapping and single key tapping [1]. The software may provide a standard way to diagnose Parkinson's, identify problems earlier in disease course (e.g. medication side effects), and track disease progression using smartphones and wearable technology. Keystroke timing information captured as patients are typing on a computer keyboard over an extended period showed that PD affects various characteristics of hand and finger movement and that these can be detected. These keystroke features can discriminate between early-PD subjects and controls with 96\% sensitivity, 97\% specificity [2]. However, it currently does not incorporate a second cardinal disease symptom, so may not differentiate PD from similar movement-related disorders.

\section{Disease Monitoring}

Patients on PD therapies are assessed using the Unified Parkinson's Disease Rating Scale part III (UPDRS-III), a compound clinical score that ascertain various motor aspects of the disease, such as rigidity, resting tremors, speech and facial expression among others. The UPDRS requires trained medical personnel and attendance of the patient in the clinic, limiting the ease and frequency with which it can be administered. Once PD patient has been started on medications, an accessible way to precisely quantify PD motor signs in the patient's home will have the potential to bring significant benefits to therapy management, better diagnosis and possibly earlier detection of the symptoms and enabling the development of new therapies. Smartphone apps and wearable 
technology with built-in sensors allow people with PD to track motor symptoms, such as tremor, as frequently as several times a day generating large amounts of data. Machine learning algorithms that use data streams captured from soft wearable sensors have the potential to automatically detect PD symptoms and inform clinicians about the progression of disease. A single wearable sensor on the back of the hand is sufficient for detecting bradykinesia and tremor in the upper extremities, whereas using sensors on both sides does not improve performance. However, these algorithms must be trained with annotated data from clinical experts who can recognize symptoms, and collecting such data is costly [3].

\section{Pre-diagnostic Phase \& Modelling the Molecular Basis}

The field of pre-diagnostic PD is fast developing with an expanding range of clinical and laboratory biomarkers, and multiple strategies seeking to discover those 'at risk' of developing PD and/or those in the earliest stages. In addition, the likelihood of securing neuroprotective benefit from PD drugs will be in these subjects, preceding current point of diagnosis of PD [4]. Current strategies to identify these individuals include disease biomarkers to demonstrate the presence of neurodegenerative synucleinopathy without clinical symptoms (Pre-clinical PD stage), and imaging markers using radiotracer imaging with single photon emission computed tomography (SPECT) or positron emission tomography (PET), and transcranial sonography (TCS) which have repeatedly demonstrated the ability to differentiate patients with PD from healthy individuals with adequate sensitivity and specificity.

Radiotracer imaging may be a good pre-diagnostic imaging marker for PD but is expensive when considering use on a large scale and further studies are required. Recent biomarker strategies have centered on finding and characterising forms of specific protein, such as $\alpha$-synuclein, in a range of biofluids and tissues. Potential CSF biomarkers for PD include $\alpha$-synuclein and DJ-1, with A 342 potentially correlating with cognitive impairment, and various forms of tau protein and neurofilament light-chain differentiating PD from atypical parkinsonian disorders [5]. However, invasiveness, cost and suitability for screening are main hurdles for adopting these strategies in every day practice. Using molecular models and simulations together with biochemical and ultrastructural analysis, researchers have shown that the clumping of $\alpha$-synuclein in the brain, in contrast to another protein, $\beta$-synuclein, which appeared to block the clumping action. Modeling the molecular basis of PD to understand the molecular mechanism for disease progression using computational modeling will have broad applicability to other diseases, but it is still in progress.

\section{Conclusion}

Applicability is the quality of being relevant or appropriate by virtue of being applicable to the matter at hand. In the last 30 years, typing cadence (known as keystroke dynamics) has been studied by various research groups and employed commercially as a biometric, mainly as a way to replace or strengthen passwords. However, applications to daily practice in the medical field are almost non-existent. Digital technologies which can objectively quantify PD motor signs have several limitations. For example, the algorithm used in the numerical neuro QWERTY index (nQi) has limited generalizability to individuals whose PD signs are not represented in the dataset, and there is always a risk of overfitting the data.

The only way to achieve full external validity, techniques such as the nQi need to be challenged in a prospective study with a large sample size that encompasses the broad spectrum of motor and non-motor characteristics that are present in a sporadic PD population (such as impaired cognition, depression and apathy). Many of the new devices and strategies, including software applications which harness information on activity and motion captured by smart phones, tablet-devices, or custom-built sensors, demonstrate high sensitivity and specificity in differentiating patients with PD from controls, and are likely to see increasing use in clinical practice to guide decision-making. Despite the indication that objective motor dysfunction occurs prior to diagnosis in PD, currently there are few examples of the application of remote or wearable devices in pre-diagnostic PD. A major foreseeable hurdle is ensuring that validation and regulatory approval for software and devices will keep abreast of rapid and continuous change in available technology.

\section{References}

1. Giancardo L, Sánchez Ferro A, Arroyo Gallego T, Butterworth I, Mendoza C S, et al. (2016) Computer keyboard interaction as an indicator of early Parkinson's disease. Scientific Reports 6: 34468.

2. Adams W R (2017) High-accuracy detection of early Parkinson's Disease using multiple characteristics of finger movement while typing. PLoS One 12(11): e0188226.

3. Lonini L, Dai A, Shawen N, Simuni T, Poon C, et al. (2018) Wearable sensors for Parkinson's disease: which data are worth collecting for training symptom detection models. npj Digital Medicine 1(64).

4. Noyce A J, Lees A J, Schrag A E (2016) The prediagnostic phase of Parkinson's disease. J Neurol Neurosurg Psychiatry 87 (80): 871-878.

5. Horak F B, Mancini M (2013) Objective biomarkers of balance and gait for Parkinson's disease using body-worn sensors. Mov Disord 28(11): 1544-1551. 